\title{
Suitable extraction conditions for determination of total anti-oxidant capacity and phenolic compounds in Ruspolia differens Serville
}

\author{
G. Ssepuuya ${ }^{1 *}$, J. Kagulire ${ }^{1}$, J. Katongole ${ }^{1}$, D. Kabbo ${ }^{1}$ J. Claes $^{2}$ and D. Nakimbugwe ${ }^{1}$ \\ ${ }^{1}$ Department of Food Technology and Nutrition, CAES, Makerere University, P.O. Box 7062, Kampala, Uganda; ${ }^{2} \mathrm{KU}$ \\ Leuven, Department of Microbial and Molecular Systems (M2S), Lab4Food, Technology Campus Geel, Kleinhoefstraat 4, \\ 2440 Geel, Belgium; gksepuya@gmail.com
}

Received: 13 April 2020 / Accepted: 6 July 2020

(c) 2020 Wageningen Academic Publishers

OPEN ACCESS C(i) ()(2) RESEARCH ARTICLE

\begin{abstract}
Recent research on edible insects' composition has placed more emphasis on the quantity and quality of macroand micro- nutrients and less emphasis on their bio-active components. This study aimed at assessing the effect of four factors, namely: defatting, solvent type (ethanol, methanol and acetone), solvent concentration (50, 75 and $100 \%)$ and extraction time (15, 30, 45 and 60 minutes) on the extraction efficiency, the total anti-oxidant activity and the amount of total phenolics and total flavonoids of Ruspolia differens. It was not known if the removal of fat (defatting) would influence the total anti-oxidant activity, and the distribution of anti-oxidant compounds in the $R$. differens matrix. Non-defatting use of acetone and sonication for 60 minutes are associated with high antioxidant activity and high amounts of bio-active compounds extracted. Aqueous acetone (50\%) extracts had the highest average anti-oxidant activity $(87.00 \pm 2.59 \%$ RSA $)$ while $100 \%$ acetone extracts had the highest average total phenolics $(11.14 \pm 0.18 \mathrm{GAE} / \mathrm{g})$ and average total flavonoids $(3.02 \pm 0.21 \mathrm{QE} / \mathrm{g})$ content, indicating the presence of other, possibly highly polar, bio-active compounds in $R$. differens.
\end{abstract}

Keywords: Ruspolia differens, anti-oxidants, defatting, extraction conditions

\section{Introduction}

Bioactive compounds are molecules that can present therapeutic potential with influence on energy intake, while reducing pro-inflammatory state, oxidative stress, and metabolic disorders (Santos et al., 2019). Many bioactive compounds possess anti-oxidant power, i.e. the capacity to scavenge free radicals, referred to as 'total anti-oxidant activity' to account for these bio-actives' combined beneficial effects on human health (Chandel et al., 2012). Anti-oxidant compounds such as phenolic compounds (including flavonoids) prevent or delay the development of chronic diseases through inhibiting mutagenesis, carcinogenesis and human heart diseases (Kiritsakis and Shahidi, 2017; Rice-Evans and Packer, 2003). Similarly, extracts of natural anti-oxidant compounds such as carotenoids, tocopherols and tocotrienols have been proven to prevent oxidative deterioration of foods such as meat (Akbarirad et al., 2016; Brewer, 2008; Haworth, 2003; Sayyari and Farahmandfar, 2017). These preventative actions against oxidative damage explain the increased interest of scientists in foods and plants with bio-active compounds that exhibit anti-oxidant activity, especially in human health related disciplines of medicine and nutrition (Aguirre and Borneo, 2013; Chandel et al., 2012; Denardin et al., 2015; Skrovankova et al., 2015).

Edible insects are important sources of protein and other nutrients (fat, minerals and vitamins) that are comparable, if not superior, to conventional foods in both quantity and quality (Rumpold and Schlüter, 2013; Ssepuuya et al., 2016). In addition, insects potentially contain bioactive compounds that are vital to human health. Edible stinkbugs and grasshoppers contain fibre (chitin), antioxidant enzymes such as superoxide dismutase, alkaloids, flavonoids, anthraquinones, tannins, phlobatannins, 
steroids, triterpenoids and cyanogenic glycosides (Musundire et al., 2014; Zielinska et al., 2017). Peptides of several edible insects including Amphiacusta annulipes, Zophobas morio and Locusta migratoria possess antioxidant activity (Denardin et al., 2015). The hydro-alcoholic extracts of the edible mole cricket (Brachytrupes orientalis) increased glucose utilisation against high glucose exposure, reduced intercellular reactive oxygen species production and reduced lipid peroxidation among other effects (Dutta et al., 2017). These findings demonstrate edible insects' anti-oxidant potential and hence possible contribution to preventing oxidative damage. Since feed influences insects' chemical (nutrient) composition, the presence of bio-active compounds in insects is possibly influenced by what they feed on (Mlcek et al., 2014). Insects such as grasshoppers and stinkbugs are herbivores, feeding on grasses and other plants, which are the major sources of anti-oxidant compounds (Agrawal and Gopal, 2013; Barbehenn et al., 2004; Behmer, 2009; Gupta et al., 2015; Musundire et al., 2016). Hence, the grasshopper Ruspolia differens can also be a potential source of anti-oxidant compounds such as catalase, glutathione, and glutathione peroxidase (Kasozi et al., 2019).

There are several methods used for identification and quantification of anti-oxidant compounds and activity in foods. The 2,2-diphenyl-1-picrylhydrazyl (DPPH) assay, Folin-Ciocalteu assay and aluminium chloride colorimetric assay are used to measure total anti-oxidant activity, total phenolic content and total flavonoids content of foods, respectively (Pisoschi and Negulescu, 2011; Prior et al., 2005). These methods are simple, both in terms of implementation and instrumentation, and are thus widely used. Despite being applied with the same principles across matrices, the extraction conditions, particularly the extraction solvent, solvent power (concentration) and extraction time, greatly vary across matrices (Agbo et al., 2015; Cosmulescu et al., 2015; Farasat and Khavari-nejad, 2014; Kodama et al., 2010; Plaza et al., 2014; Ramamoorthy and Bono, 2007; Rebaya et al., 2014). To various extents, these conditions have been modified and used in studies for identification and quantification of anti-oxidant compounds and activity of hundreds of foods of plant origin especially fruits, vegetables and cereals (Liu et al., 2014; Moyo et al., 2013; Stangeland et al., 2009).

Information on the presence and quantities of anti-oxidant compounds and activity in edible insects such as $R$. differens can hardly be found in published literature. Additionally, information on the most suitable conditions for extraction, identification and quantification of anti-oxidant compounds in $R$. differens, including the possibility of defatting prior to extraction, is lacking. Removal of fat from the matrix (defatting) is necessary to determine as to whether a substantial amount of the anti-oxidant compounds are preferentially found in the apolar fat phase of the
R. differens matrix or not. In the former case, they could have an important role to play in the oxidative stability of the highly fatty $R$. differens. This study therefore aimed at (1) establishing the most efficient conditions (i.e. conditions that result in the highest values) for anti-oxidant extraction and quantification including defatting; (2) determining the total anti-oxidant activity; and (3) quantifying the total phenolics and total flavonoids content of defatted and non-defatted $R$. differens.

\section{Materials and methods}

\section{Sampling and sample preparation}

Fresh plucked (with wings, legs and antennae removed) Ruspolia differens $(5 \mathrm{~kg})$ was collected from the seasonal grasshopper roadside market in Nyendo, central region of Uganda, about $115 \mathrm{~km}$ southwest of Kampala along Masaka-Kampala Road ( $0^{\circ} 18^{\prime} 32.37^{\prime \prime}$ '; 31 $41^{\circ} 47.19^{\prime \prime E}$, 1261 meters above sea level). Nyendo was chosen because it doubles as a major catching area and major market, receiving grasshoppers from nearby catching areas and supplying them to other markets, especially Kampala, the capital city of Uganda. They were washed clean, drained, packed in polyethene bags and stored at $-18^{\circ} \mathrm{C}$. On the day of analysis, $30 \mathrm{~g}$ of the frozen $R$. differens was thawed at $5-7^{\circ} \mathrm{C}$ for 2 hours and pulverised into a fine paste using a hand-held blender (600 W SilverCrest blender; Kompernaß Handelsgesellschaft mbH, Bochum, Germany) operated at speed 4 for 3 minutes. This was the non-defatted $R$. differens sample. A $10 \mathrm{~g}$ portion of this paste was defatted using the Folch method (Wrolstad et al., 2005). In brief, $100 \mathrm{~g}$ of raw $R$. differens paste was mixed with $300 \mathrm{ml}$ of 2:1 chloroform (SUVCHEM, Mumbai, India) : methanol (Loba Chemie Pvt. Ltd., Mumbai, India) mixture, i.e. the fat extraction solvent mixture. The mixture was hand-shaken vigorously for five minutes at room temperature and filtred by suction into a clean 11 flask through a Whatman no. 1 filter (Sigma Aldrich, St. Louis, MO, USA). The residue was further extracted twice using $200 \mathrm{ml}$ of the fat extraction solvent mixture each time. The oil containing filtrate was discarded and the residue was retained as the defatted $R$. differens sample. Aliquots were drawn from both the defatted and the non-defatted $R$. differens for extraction and subsequent determination of the total anti-oxidant activity, and quantification of total phenolics and total flavonoids.

\section{Extraction of bio-active compounds}

Prior to extraction, bio-actives' extraction solvent mixtures were made by mixing each of the pure organic solvents, i.e. ethanol (VWR International, Fontenay Sous Bois, France), methanol (Loba Chemie Pvt. Ltd.) or acetone (Labo Chemie Pvt. Ltd., Mumbai, India) with distilled water in rations of 50:50, 75:25 and 100:0, organic solvent/distilled water, v/v, 
resulting in solutions with 50,75 and $100 \%$ of the organic solvent. From the non-defatted and defatted $R$. differens samples, bio-actives were extracted in triplicate as described by Wrolstad et al. (2005). Briefly, $1.0 \mathrm{~g}$ of sample (nondefatted or defatted $R$. differens) was weighed to the nearest $0.01 \mathrm{~g}$ into a $15 \mathrm{ml}$ test tube and mixed with $5 \mathrm{ml}$ of the bio-actives' extracting solvent mixture of either 50,75 or $100 \%$ of organic solvent by volume. Under subdued light at room temperature $\left(\sim 25^{\circ} \mathrm{C}\right)$, the samples were extracted by ultra-sonication in a sonicator (Bransonic series, M 2800-E; Branson Ultrasonics Co, Danbury, CT, USA) for either 15, 30, 45 or 60 minutes with periodic shaking. The homogenate was cooled to $\leq 4{ }^{\circ} \mathrm{C}$ and centrifuged (model 225; Thermo Fisher Scientific Inc, Merelbeke, Belgium) at $4550 \mathrm{rpm}$ for 10 minutes. The supernatant was decanted, stored below $4{ }^{\circ} \mathrm{C}$ and the residue re-extracted as described above. The supernatants from both extractions were combined in a $10 \mathrm{ml}$ volumetric flask, made up to the $10 \mathrm{ml}$ mark with the extracting solvent mixture used, centrifuged at 4,550 rpm for 10 minutes, immediately cooled and stored below $4{ }^{\circ} \mathrm{C}$ for further analysis.

\section{Total anti-oxidant activity}

This was determined by the DPPH assay (Diem Do et al., 2014; Thaipong et al., 2006). Accurately, $0.1 \mathrm{ml}$ of a sample extract (section 'Extraction of bio-active compounds') or $0.1 \mathrm{ml}$ of the extracting solvent (control) was each mixed with $2.9 \mathrm{ml}$ of the DPPH working solution (3.9 mg of DPPH; Sigma Aldrich, in $100 \mathrm{ml}$ methanol; Loba Chemie Pvt.). The mixtures were vortexed for 30 seconds and incubated for 30 minutes in the dark at room temperature. The absorbances (ABS) of the blank (control) and sample extracts were measured at 517 nm using a UV spectrophotometer (GENESYS $10 \mathrm{UV}$; Thermal Electron Corporation, Masdon, OH, USA). The total anti-oxidant capacity of each of the samples was expressed as percentage radical scavenging capacity (\% RSA) from the ABS measurements according to Equation 1. All determinations were done in triplicate.

Percentage radical scavenging capacity (\% RSA) =

$\mathrm{ABS}_{\text {control }}-\frac{\mathrm{ABS}_{\text {sample }}}{\mathrm{ABS}_{\text {control }}}$

\section{Total phenolics}

This was assayed by the Folin-Ciocalteu method (Diem Do et al., 2014; Thaipong et al., 2006). Three different solutions were prepared: the sample under study (section 'Extraction of bio-active compounds'), garlic acid (Sigma Aldrich) as the calibration standard and a blank (distilled water). For each solution, $0.3 \mathrm{ml}$ was mixed, under subdued light, with $3 \mathrm{ml}$ of distilled water, $0.3 \mathrm{ml}$ of $0.25 \mathrm{M}$ FolinCiocalteu (Sigma Aldrich) reagent (3.125 $\mathrm{ml}$ of stock in
$25 \mathrm{ml}$ of distilled water) and $0.6 \mathrm{ml}$ of 1 molar sodium carbonate (UNILAB, Nairobi, Kenya) solution, were added in that order. The volume of each mixture was made up to $5 \mathrm{ml}$ with the extracting solvent mixture used (section 'Extraction of bio-active compounds'). The mixtures were vortexed and incubated for 40 minutes at room temperature $\left(\sim 25^{\circ} \mathrm{C}\right)$. After incubation, the absorbance of the sample extract, calibration standard and blank was measured at $765 \mathrm{~nm}$ in a $1 \mathrm{ml}$ glass cuvette using a UV spectrophotometer (GENESYS 10 UV; Thermal Electron Corporation). The concentration of the sample extract $(\mathrm{GAE} / \mathrm{ml})$ was obtained from the garlic acid (Sigma Aldrich) calibration curve (Supplementary Figure S1). To prepare the calibration curve, a stock solution of $1 \mathrm{mg}$ of garlic acid per $\mathrm{ml}$ was prepared. From this stock solution, calibration solutions of $0.025,0.050,0.075,0.100,0.125$ and $0.150 \mathrm{mg} / \mathrm{ml}$ were prepared and there absorbances were measured and used to prepare the calibration curve in Supplementary Figure $\mathrm{S} 1$. Total phenolics was expressed as garlic acid equivalents (GAE) per g of $R$. differens using Equation 2 below. All determinations were done in triplicate.

$\mathrm{GAE} / \mathrm{g}=\frac{\mathrm{GA}}{\mathrm{ml}} \times \frac{10 \mathrm{ml}}{\mathrm{W}_{\mathrm{g}}}$

Where $\mathrm{GA} / \mathrm{ml}$ is the concentration from the calibration curve, $10 \mathrm{ml}$ is the final volume of the sample extract (section 'Total anti-oxidant activity'), and $\mathrm{W}_{\mathrm{g}}$ is the weight of the sample that was used to prepare the $10 \mathrm{ml}$ sample extract.

\section{Total flavonoids}

This was assayed by the aluminium chloride colorimetric method (Diem Do et al., 2014; Thaipong et al., 2006). Three different solutions were prepared: the sample under study (section 'Extraction of bio-active compounds'), quercetin (Sigma Aldrich) as the calibration standard and a blank with distilled water. For each solution, (1) $1.0 \mathrm{ml}$ of the sample extract (section 'Extraction of bioactive compounds'); (2) $1.0 \mathrm{ml}$ of quercetin (Sigma Aldrich) calibration standard (Supplementary Figure S2); and (3) $1.0 \mathrm{ml}$ of the blank (distilled water), under subdued light at room temperature $\left(\sim 25^{\circ} \mathrm{C}\right)$, was mixed with $0.2 \mathrm{ml}$ of $5 \% \mathrm{NaNO}_{2}$ (Loba Chemie Pvt. Ltd.) (w/v) and the mixture incubated for 5 minutes. To each solution after incubation, $0.2 \mathrm{ml}$ of $10 \% \mathrm{AlCl}_{3}$ (Sigma Aldrich) (w/v) was added, each solution incubated for 6 minutes after which $2 \mathrm{ml}$ of $1 \mathrm{M}$ $\mathrm{NaOH}$ (Loba Chemie Pvt. Ltd.) solution was added. The volume of each mixture was made up to $5 \mathrm{ml}$ with the extracting solvent mixture used (section 'Extraction of bioactive compounds') and the absorbance of each solution measured at $510 \mathrm{~nm}$ using a UV spectrophotometer (GENESYS 10 UV; Thermal Electron Corporation). The concentration of the sample extract $(\mathrm{QE} / \mathrm{ml})$ was read from a quercetin calibration curve (Supplementary Figure S2). To prepare the calibration curve, a stock solution of $4 \mathrm{mg}$ 
of quercetin per $\mathrm{ml}$ was prepared. From this stock solution, calibration solutions of $0.4,0.8,1.2,1.6,2.0,2.4,2.8,3.2$, 3.6 and $4.0 \mathrm{mg} / \mathrm{ml}$ were prepared and there absorbances were measured and used to prepare the calibration curve in Supplementary Figure S2. Total flavonoids were expressed as quercetin equivalents (QE) per $g$ of $R$. differens according to Equation 3 below. All determinations were done in triplicate.

$\mathrm{QE} / \mathrm{g}=\frac{\mathrm{Q}}{\mathrm{ml}} \times \frac{10 \mathrm{ml}}{\mathrm{W}_{\mathrm{g}}}$

Where $\mathrm{Q} / \mathrm{ml}$ is the concentration from the calibration curve, $10 \mathrm{ml}$ is the final volume of the sample extract (section 'Total anti-oxidant activity'), and $\mathrm{W}_{\mathrm{g}}$ is the weight of the sample that was used to prepare the $10 \mathrm{ml}$ sample extract.

\section{Statistical procedure}

Statistical Package for Social Scientists (SPSS) for Windows (Version 20; IBM Corporation, Armonk, NY, USA) was used for data analysis. Descriptive statistics were used to obtain means and standard deviations. The data had a normal distribution and the ANOVA test was used to determine the effect of defatting, solvent, solvent power and sonication time on the extraction efficiency of total anti-oxidants, total phenolics and total flavonoids of $R$. differens. Means were separated by the Turkey's option of the ANOVA test. The graphs are presented for easy visibility of the results while Supplementary Table S1 and S2 show the statistical differences

\section{Results}

\section{Defatting}

Defatting (removing fat from the $R$. differens matrix) leads to a consistent significant $(P<0.05)$ reduction in total antioxidant activity, and the amounts of total phenolics and total flavonoids at $75 \%$ and $100 \%$ solvent concentrations, except for $50 \%$ organic solvent concentration where the trend is not consistent. The reduction is more consistent at higher ultra-sonication times (45 and 60 minutes).

\section{Solvent type}

Three solvents, i.e. ethanol, methanol and acetone were used. At $50 \%$ solvent concentration, acetone extracts exhibit the highest total anti-oxidant activity, significantly different $(P \leq 0.05)$ from methanol and comparable to ethanol extracts especially for the non-defatted $R$. differens sample (Figure 1 , Supplementary Table S1). Acetone extracts show the highest amounts of total phenolics, significantly $(P<0.05)$ higher than the amounts from methanol and ethanol extracts especially for non-defatted $R$. differens (Figure 2, Supplementary Table S1 and S3). For defatted R. differens, the amount of total phenolics in acetone extracts was either significantly higher or equal to that in methanol and ethanol extracts. Non-defatted $R$. differens acetone extracts have significantly $(P<0.05)$ higher amounts of total flavonoids than ethanol and methanol extracts (Figure 3, Supplementary Table S1). The amount of total flavonoids in ethanol extracts of defatted $R$. differens was either equal or higher than that of methanol extracts (Figure 3, Supplementary Table S1).

\section{Solvent concentration}

At each ultra-sonication time and solvent type for both defatted and non-defatted samples, solvent concentration significantly $(P<0.05)$ influences the total anti-oxidant activity, and the amounts of total phenolics and total flavonoids extracted from $R$. differens, except for the defatted ethanol extract at 15 minutes $(P=0.261)$ (Supplementary Table S2). For all the solvents and ultra-sonication times, $50 \%$ solvent power extracts significantly had the highest total anti-oxidant activity $(54.71 \pm 0.80-87.00 \pm 2.59 \%$ RSA $)$, followed by $75 \%(27.39 \pm 1.76-74.37 \pm 1.77 \%$ RSA $)$ and lastly, $100 \%$ which had the lowest (5.22 $\pm 2.33-38.91 \pm 4.36 \%$ RSA) (Figure 1). Except for the non-defatted $R$. differens acetone extracts that exhibited the highest total phenolics content $(10.10 \pm 0.39-11.14 \pm 0.18 \%)$ at $100 \%$ solvent concentration and at all the sonication times, the amounts of total phenolics extracted decreased with increasing solvent concentration, though not always significantly $(P<0.05)$, for every solvent and ultra-sonication time (Figure 2, Supplementary Table S2). Though the trend is unclear, especially for the defatted $R$. differens, the amounts of total flavonoids extracted by $100 \%$ ethanol and acetone from the non-defatted $R$. differens were significantly higher than those at lower concentrations. One hundred percent acetone extracted the highest amounts of total flavonoids from the $R$. differens matrix while $100 \%$ methanol extracted the lowest amounts (Figure 3, Supplementary Table S2 and S3).

\section{Ultrasonication time}

For each sample type, solvent type and solvent concentration, there was in general, though not always, a significant increase in the total anti-oxidant activity (\% RSA) with an increase in ultra-sonication time (Figure 1, Supplementary Table S2), with the highest values obtained at 60 minutes. There is no clear trend regarding total phenolics and total flavonoids content of ethanol and methanol extracts with changes in ultra-sonication time for defatted $R$. differens. Non-defatted $R$. differens' samples ultra-sonicated for 45 and 60 minutes exhibited slightly higher amounts of total phenolics than those ultra-sonicated for 15 and 30 minutes (Figure 2, Supplementary Table S2). At 100\% organic solvent concentration, the total flavonoids content of non-defatted $R$. differens increases with an increase in 


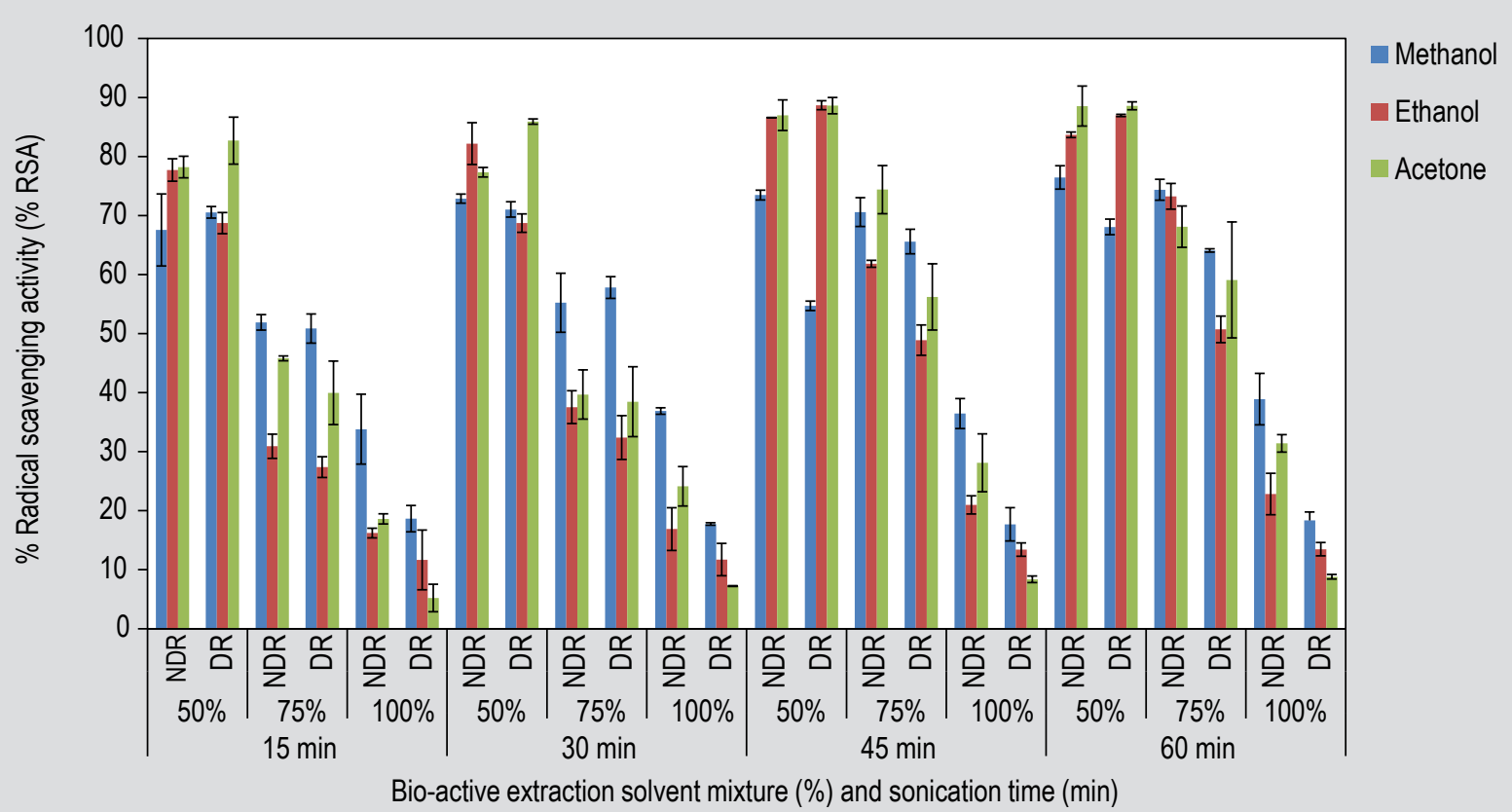

Figure 1. The effect of defatting, bio-active extraction solvent mixture (\%) and sonication time (minutes) on the total antioxidant activity of Ruspolia differens (NDR = non-defatted $R$. differens; DR = defatted $R$. differens; $\%=$ percentage of the organic solvent in the bio-active extraction solvent mixture, the other part being distilled).

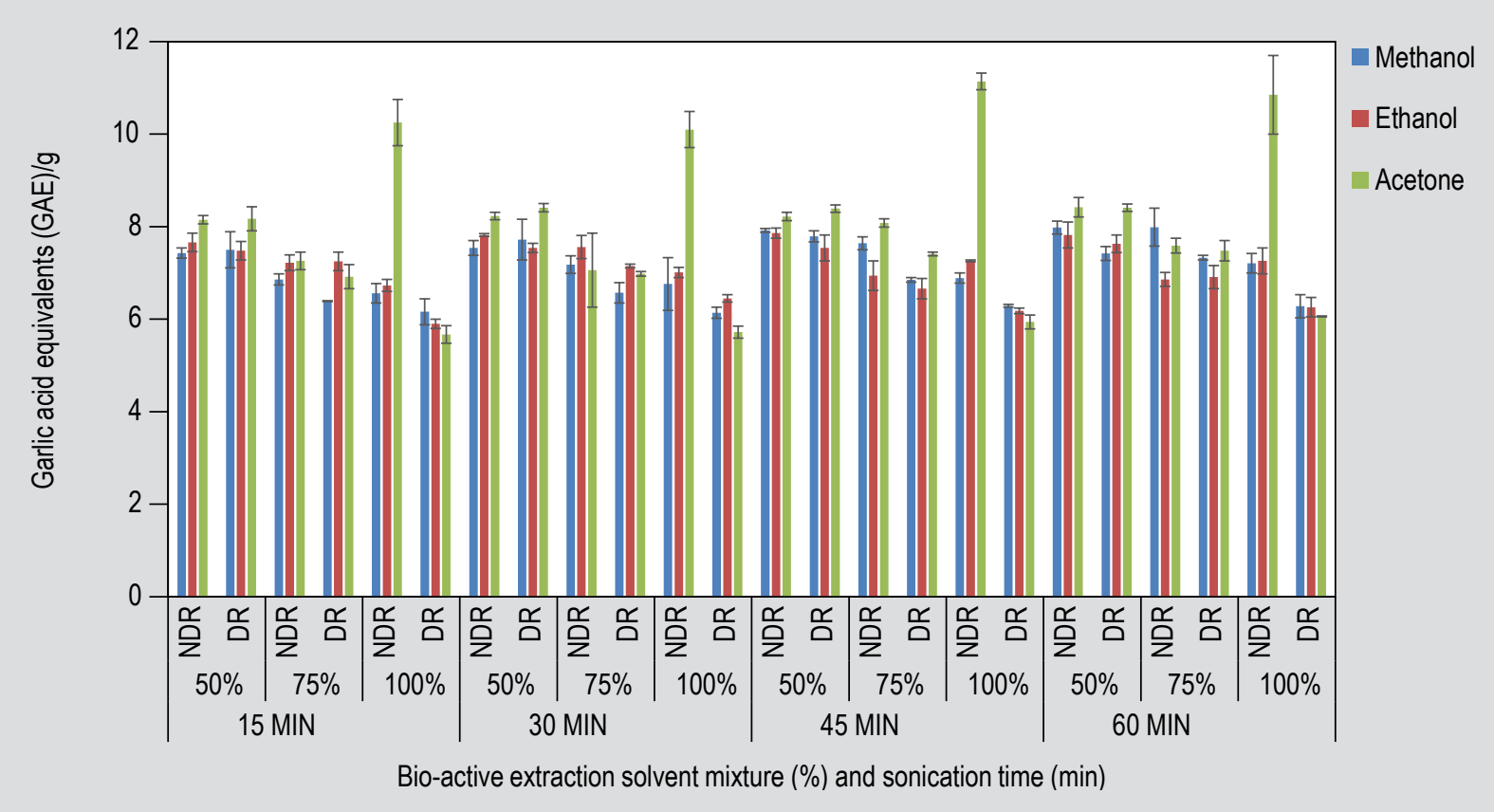

Figure 2. The effect of defatting, bio-active extraction solvent mixture (\%) and sonication time (minutes) on the total phenolics' content of Ruspolia differens. (NDR = non-defatted $R$. differens; $\mathrm{DR}=$ defatted $R$. differens; $\%=$ percentage of the organic solvent in the bio-active extraction solvent mixture, the other part being distilled).

ultra-sonication time. The trend is however inconsistent for $50 \%$ and $75 \%$ organic solvent extracts. Though not always, $R$. differens ultra-sonicated for 45 and 60 minutes had slightly $(P<0.05)$ higher amounts of total flavonoids than those ultra-sonicated for 15 and 30 minutes (Figure 3, Supplementary Table S2 and S3). 


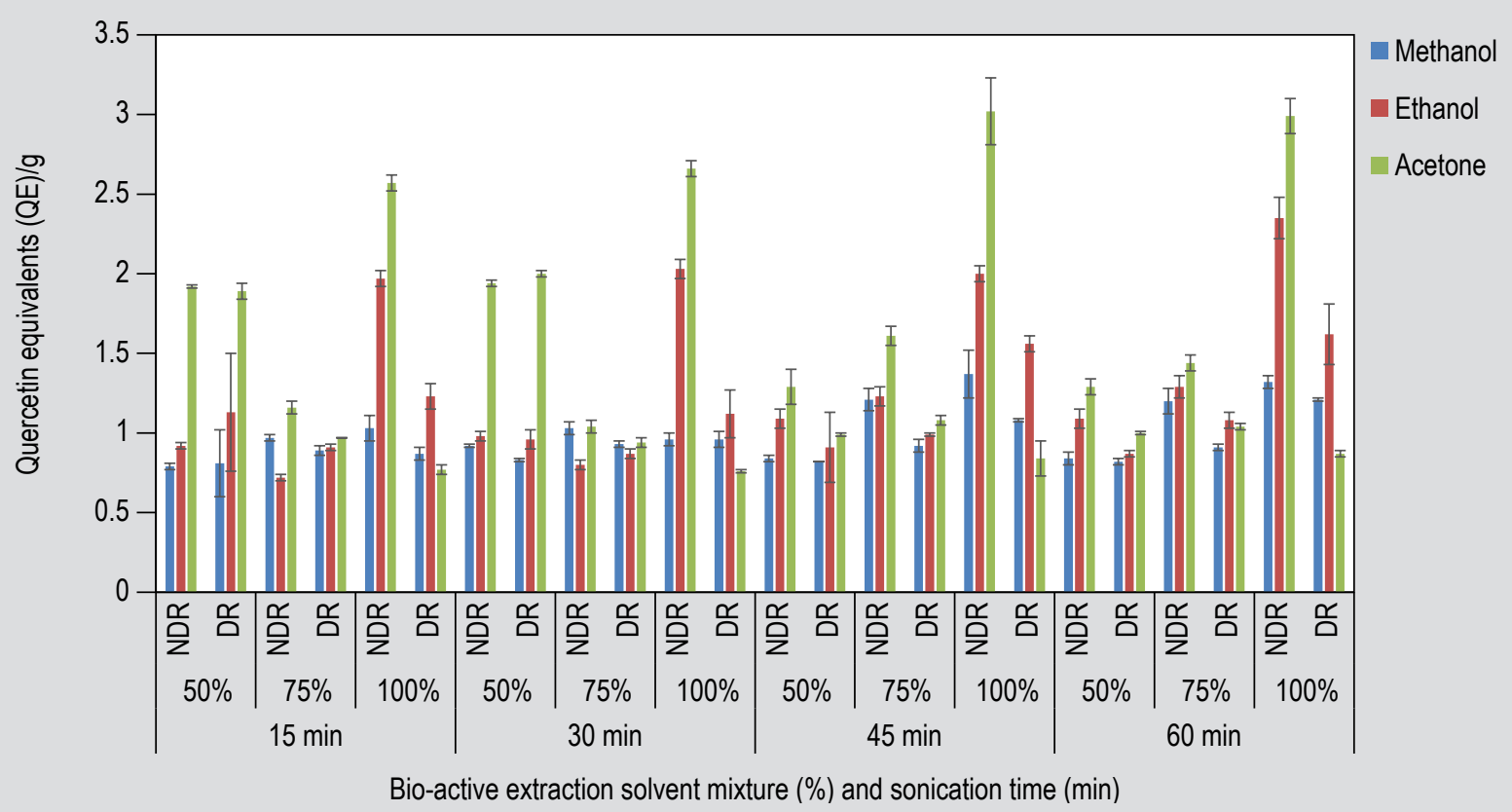

Figure 3. The effect of defatting, bio-active extraction solvent mixture (\%) and sonication time (minutes) on the total flavonoids' content of Ruspolia differens. (NDR = non-defatted $R$. differens; DR = defatted $R$. differens; $\%=$ percentage of the organic solvent in the bio-active extraction solvent mixture, the other part being distilled).

\section{Discussion}

The highest total anti-oxidant activity, total phenolics content and total flavonoids content were obtained using acetone as a solvent. Similarly, in several other studies, acetone at different concentrations was the best solvent for extraction of anti-oxidants, especially from matrices of plant origin (Boeing et al., 2014; Dent et al., 2013; Drużyńska et al., 2007; Quispe-fuentes et al., 2017). The solubility and extractability of anti-oxidant compounds such as polyphenols depends on the polarity of the extracting solvent (Quispe-fuentes et al., 2017). Among the solvents used in this study, methanol has the highest polarity followed by ethanol, with acetone being the least polar. This indicates preference of phenolic and flavonoid antioxidant compounds in the $R$. differens matrix for a less polar solvent. Under the extraction conditions used in this study, acetone is better at solubilising the anti-oxidant compounds in $R$. differens.

Besides the solvent, its power (concentration) affects the yield and activity of anti-oxidant compounds (Ballesteros et al., 2014). Pure solvent (100\%) extracts exhibited either equal or the highest amounts of total phenolics and total flavonoids especially for non-defatted $R$. differens compared to 50 and $75 \%$ solvent concentrations. Similar observations have been made in non-insect samples such as the Limnophila aromatica, a spice and a medicinal herb in Southeast Asia (Diem Do et al., 2014). To the contrary, the highest total anti-oxidant activity was observed with a more polar organic solvent, that is $50 \%$ mixtures of the solvent with water, i.e. $50 \%$ acetone and $50 \%$ ethanol extracts. Given that: (1) not all compounds with anti-oxidant activity in the $R$. differens matrix were identified and quantified; and (2) total anti-oxidant activity is a measure of all compounds with anti-oxidant activity (Pisoschi and Negulescu, 2011), it is possible that the other unidentified compounds such as anti-oxidant proteins and vitamins ( $\mathrm{C}$ - and the $\mathrm{B}$-complex) (Feng et al., 2017; Higashi-Okai et al., 2006; McDowell et al., 2007) required a more polar solvent for extraction and thus, subsequent expression of anti-oxidant activity. One study in insects (Musundire et al., 2014) and numerous studies in plant matrices reported aqueous mixtures of different solvents as having the highest anti-oxidant yield and activity (Addai et al., 2013; Boeing et al., 2014; Moyo et al., 2013; Qasim, 2016). Also, in studies where $100 \%$ organic solvent suitably extracted antioxidant compounds such as phenols and flavonoids, the highest total anti-oxidant yield and activity was observed with aqueous solvent (5080\% V/V solvent/water) extracts (Diem Do et al., 2014; Syukriah, 2014). This is because of the possible presence of various anti-oxidant compounds with different chemical characteristics and polarities (Diem Do et al., 2014).

The duration of extraction influences the yield of antioxidant compounds and total anti-oxidant activity (Annegowda et al., 2012; Butsat and Siriamornpun, 2016; Diem Do et al., 2014). In this study, the total anti-oxidant activity, and the amounts of total phenolics content and total carotenoid were higher at 45 and 60 minutes of 
sonication, than at 15 and 30 minutes. However, to extract bio-active compounds from edible stinkbugs (Encosternum delegorguei) consumed in south-eastern districts of Zimbabwe, 10 minutes (without optimisation) were used (Musundire et al., 2014). This study and that of Musundire et al. (2014), imply that the extraction time of anti-oxidant compounds is matrix dependent and should be validated for every matrix to avoid underestimation of anti-oxidant compounds and anti-oxidant activity.

The maximum total anti-oxidant activity observed $(87.00 \pm 2.59 \%, 50 \%$ acetone extract) is comparable to that of $50 \%$ methanolic extract of raw edible stinkbugs

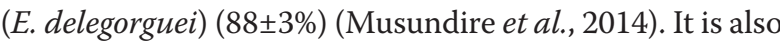
comparable to that observed in commonly consumed fruits and vegetables. For example, the highest total anti-oxidant activity $(87.00 \pm 2.59 \%$ RSA) was similar to that of many fruits and vegetables commonly consumed in china, many of which, such as cabbages and eggplants are part of African diets, except for plums, jujube, chilli pepper and the Chinese toon whose values are higher (Ramamoorthy and Bono, 2007). The total flavonoids content observed was higher than that found in 16 fruits and vegetables consumed in Burkina Faso (0.22-3.34 GAE/g) (Bayili et al., 2011). The total phenolic and total flavonoids content was respectively similar to approximately $90 \%$ of the fruits and vegetables commonly consumed in China (Liu et al., 2014). Hence, the total anti-oxidant capacity, total phenolics content and total flavonoids content of $R$. differens was similar to that of many good fruit and vegetable sources of antioxidant compounds.

Although $R$. differens is consumed whole, the possibilities of extracting nutrient components and using the extracted components as ingredients in various foods or other products exist. Ruspolia differens contains approximately 42.21-54.33\% fat and 34.23-45.76\% protein, the rest being chitin, carbohydrate and fibre. Fat and non-fat components can be extracted and used as ingredients. Presence of antioxidant compounds in the oil/fat phase can protect the oil from oxidative rancidity while the presence of these compounds in the non-fat phase enhances the latter's nutrient composition. The non-defatted $R$. differens showed the highest total anti-oxidant activity, total flavonoids content and total phenolics content irrespective of the levels of the other factors. It is therefore likely that during defatting prior to extraction (section 'Sampling and sample preparation'), the defatting solvents dissolve a portion of the anti-oxidant compounds, leading to reduced amounts of anti-oxidant compounds and hence reduced total anti-oxidant activity in the defatted sample. This is possibly because methanol, used as part of the 2:1 chloroform/methanol mixture for fat extraction, has the capacity to extract phytochemicals from a wide array of sources (Apak et al., 2013; Brewer, 2008), including $R$. differens as shown in this research. However, the total anti-oxidant activity values of the defatted samples were similar to those of the non-defatted $R$. differens sample. This implies that the anti-oxidant compounds in $R$. differens are mostly associated with the (polar) non-fat components and not the (apolar) fat, and hence may not play a major role towards preventing oxidative rancidity of the apolar phase, i.e. the $R$. differens' fat. This is an important observation that requires further research.

\section{Conclusions and recommendations}

The study aimed at establishing suitable conditions for extraction of anti-oxidant compounds from $R$. differens, including defatting. It was also not clear if the latter affects the distribution of anti-oxidant compounds between the polar and apolar (fat) phase of the $R$. differens matrix. This study found non-defatting, use of acetone and sonication for 60 minutes as the most suitable conditions. Though defatting leads to a slight reduction in percentage total anti-oxidant activity, total phenolics and total flavonoids content due to the solvents used in the defatting step, the amounts in the defatted and non-defatted $R$. differens remain similar. This implies that anti-oxidant compounds are predominantly found in the apolar non-fat phase and the defatting step is not necessary. Aqueous acetone and ethanol (50\%) extracts showed the highest total anti-oxidant activity while $100 \%$ acetone extracts had the highest total phenolics and flavonoids content, implying the existence other polar anti-oxidant compounds that weren't quantified. The highest concentration of anti-oxidant compounds was obtained at after sonication for 60 minutes. Ruspolia differens' total anti-oxidant activity and the amounts of total phenolics and flavonoids are relatively high and comparable to those of many fruits and vegetables. The high $R$. differens' anti-oxidant activity (\% RSA) indicates the presence of either, a wide variety of anti-oxidant compounds, a high amount of a few anti-oxidant compounds or a small amount of highly effective anti-oxidant compounds. Research into identification, quantification and activity of bio-active compounds in $R$. differens and other edible insects is therefore very essential.

\section{Acknowledgements}

FWO, the 'Research Foundation of Flanders' is highly acknowledged.

\section{Conflict of interest}

The authors declare that there is no conflict of interest.

\section{Supplementary material}

Supplementary material can be found online at https://doi. org/10.3920/JIFF2020.0028. 
Figure S1. Calibration curve for quantification of total phenolics.

Figure S2. Calibration curve for quantification of total flavonoids.

Table S1. Effect of defatting and solvent type on TAA, total phenolics and total flavonoid content of Ruspolia differens.

Table S2. Effect of solvent power and extraction (ultrasonication) time on TAA, total phenolics and total flavonoid content of Ruspolia differens.

Table S3. $P$-values for $t$-tests and post-hoc tests.

\section{References}

Addai, Z.R., Abdullah, A. and Mutalib, S.A., 2013. Effect of extraction solvents on the phenolic content and antioxidant properties of two papaya cultivars. Journal of Medicinal Plants Research 7: 3354-3359. https://doi: 10.5897/JMPR2013.5116

Agbo, M.O., Uzor, P.F., Akazie-Nneji, N.U., Eze-Odurukwe, C.U., Ogbatue, U.B. and Mbaoji, E.C., 2015. Antioxidant, total phenolic and flavonoid content of selected Nigerian medicinal plants. Dhaka University Journal of Pharmaceutical Sciences 14: 34-41. https:// doi.org/10.3329/dujps.v14i1.23733

Agrawal, A. and Gopal, K., 2013. Biomonitoring of water and waste water. Springer, New Delhi, India, 166 pp. https://doi. org/10.1007/978-81-322-0864-8_6

Aguirre, A. and Borneo, R., 2013. Antioxidant capacity of medicinal plants. In: Watson, R.R. and Preedy, V.R. (eds.) Bioactive food as dietary interventions for liver and gastrointestinal disease. Academic Press, Cambidge, MA, USA, pp. 527-534.

Akbarirad, H., Ardabili, G., Kazemeini, S.M. and Khaneghah, M., 2016. An overview on some of important sources of natural antioxidants. International Food Research Journal 23: 928-933.

Annegowda, H.V., Bhat, R. and Min-tze, L., 2012. Influence of sonication treatments and extraction solvents on the phenolics and antioxidants in star fruits. Journal of Food Science and Technology 49: 510-514. https://doi: 10.1007/s13197-011-0435-8

Apak, R., Gorinstein, S., Böhm, V., Schaich, K.M., Özyürek, M. and Güçlü, K., 2013. Methods of measurement and evaluation of natural antioxidant capacity/activity (IUPAC technical report). Pure and Applied Chemistry 85: 957-998. http://dx.doi.org/10.1351/PACREP-12-07-15

Ballesteros, L.F., Teixeira, J.A. and Mussatto, S.I., 2014. Selection of the solvent and extraction conditions for maximum recovery of antioxidant phenolic compounds from coffee silverskin. Food and Bioprocess Technology 7: 1322-1332. http://doi 10.1007/s11947013-1115-7

Barbehenn, R.V, Karowe, D.N. and Chen, Z., 2004. Plant animal interactions performance of a generalist grasshopper on a C3 and a $\mathrm{C} 4$ grass: compensation for the effects of elevated $\mathrm{CO}_{2}$ on plant nutritional quality. Oecologia 140: 96-103. https://doi.org/10.1007/ s00442-004-1555-x
Bayili, R.G., Abdoul-latif, F., Kone, O.H., Diao, M., Imael, H., Bassole, N. and Dicko, M.H., 2011. Phenolic compounds and antioxidant activities in some fruits and vegetables from Burkina Faso. African Journal of Biotechnology 10: 13543-13547.

Behmer, S.T., 2009. Insect herbivore nutrient regulation. Annual Review of Entomology 54: 165-187. http://doi.org/10.1146/annurev. ento.54.110807.090537

Boeing, J.S., Barizão, É.O., Costa, B. and Montanher, P.F., 2014. Evaluation of solvent effect on the extraction of phenolic compounds and antioxidant capacities from the berries: application of principal component analysis. Chemistry Central Journal 8: 1-9.

Brewer, S., 2008. Preserving beef quality with natural antioxidants. Cattlemen's Beef Board and National Cattlemen's Beef Association, Washington, DC, USA. Available at: https://tinyurl.com/y69g575w

Butsat, S. and Siriamornpun, S., 2016. Effect of solvent types and extraction times on phenolic and flavonoid contents and antioxidant activity in leaf extracts of Amomum chinense C. International Food Research Journal 23: 180-187.

Chandel, M., Sharma, U., Kumar, N., Singh, B. and Kaur, S., 2012. Antioxidant activity and identification of bioactive compounds from leaves of Anthocephalus cadamba by ultra-performance liquid chromatography/electrospray ionization quadrupole time of flight mass spectrometry. Asian Pacific Journal of Tropical Medicine 5: 977-985

Cosmulescu, S., Trandafir, I., Nour, V. and Botu, M., 2015. Total phenolic, flavonoid distribution and antioxidant capacity in skin, pulp and fruit extracts of plum cultivars. Journal of Food Biochemistry 39: 64-69. https://doi.org/10.1111/jfbc.12112

Denardin, C.C., Hirsch, G.E., Da Rocha, R.F., Vizzotto, M., Henriques, A.T., Moreira, J.C.F., Guma, F.T.C.R. and Emanuelli, T., 2015. Antioxidant capacity and bioactive compounds of four Brazilian native fruits. Journal of Food and Drug Analysis 23: 387-398. https:// doi.org/10.1016/j.jfda.2015.01.006

Dent, M., Dragovi, V., Peni, M. and Brn, M., 2013. The effect of extraction eolvents, temperature and time on the composition and mass fraction of polyphenols in Dalmatian wild sage (Salvia officinalis L.) extracts. Food Technology and Biotechnology 51: 84-91.

Diem Do, Q., Angkawijaya, A.E., LanTran-Nguyen, P., Huynh, L.H., EdiSoetaredjo, E.F., Ismadji, S. and Ju, Y.-H., 2014. Effect of extraction solvent on total phenol content, total flavonoid content, and antioxidant activity of Limnophila aromatica. Journal of Food and Drug Analysis 22: 296-302. https://doi.org/10.1016/j. jfda.2013.11.001

Drużyńska, B., Stępniewska, A. and Wołosiak, R., 2007. The influence of time and type of solvent on efficiency of the extraction of polyphenols from green tea and antioxidant properties obtained extracts. Acta Scientiarum Polonorum Technologia Alimentaria 6: 27-36.

Dutta, P., Dey, T., Dihingia, A., Manna, P. and Kalita, J., 2017. Antioxidant and glucose metabolizing potential of edible insect, Brachytrupes orientalis via modulating Nrf2/AMPK/GLUT4 signaling pathway. Biomedicine \& Pharmacotherapy 95: 556-563. https://doi.org/10.1016/j.biopha.2017.08.094 
Farasat, M. and Khavari-nejad, R., 2014. Antioxidant activity, total phenolics and flavonoid contents of some edible green seaweeds from northern coasts of the Persian gulf. Iranian Journal of Pharmaceutical Research 13: 163-170.

Feng, P., Ding, H., Lin, H. and Chen, W., 2017. AOD: the antioxidant protein database. Scientific Reports 7: 7449. https://doi.org/10.1038/ s41598-017-08115-6.

Gupta, V.K., Tuohy, M.G., O'Donovan, A. and Lohani, M., 2015. Biotechnology of bioactive compounds: sources and applications. John Wiley \& Sons, Chichester, UK, 736 pp.

Haworth, J.E., 2003. Natural antioxidants review. In: Proceedings of the $56^{\text {th }}$ American Meat Science Association Reciprocal Meat Conference. June 15-18, 2003. Columbia, MO, USA, pp. 95-98. Available at: https://tinyurl.com/yytoze9h

Higashi-Okai, K., Nagino, H., Yamada, K. and Okai, Y., 2006. Antioxidant and prooxidant activities of $\mathrm{B}$ group vitamins in lipid peroxidation. Journal of UOEH 28: 359-368. https://doi.org/10.7888/ juoeh.28.359

Kasozi, K.I., Namazi, C., Basemera, E., Atuheire, C., Odwee, A., Majalija, S. and Kateregga, J.N., 2019. Inorganic pollutants in edible grasshoppers (Ruspolia nitidula) of Uganda and their major public health implications. African Health Sciences 19: 2679-2691. https:// doi.org/10.4314/ahs.v19i3.44

Kiritsakis, A.K. and Shahidi, F., 2017. Olives and olive oil as functional foods: bioactivity, chemistry and processing. John Wiley \& Sons, Hoboken, NJ, USA, 688 pp.

Kodama, D.H., Elisa, A., Schmidt, D.S., Lajolo, F.M. and Genovese, M.I., 2010. Flavonoids, total phenolics and antioxidant capacity: comparison between commercial green tea preparations. Ciência e Tecnologia de Alimentos 30: 1077-1082.

Liu, C., Zhao, Y., Li, X., Jia, J., Chen, Y. and Hua, Z., 2014. Antioxidant capacities and main reducing substance contents in 110 fruits and vegetables eaten in China. Food and Nutrition Sciences 5: 293-307. https://doi.org/10.4236/fns.2014.54036

McDowell, L., Wilkinson, N., Madison, R. and Felix, T., 2007. Vitamins and minerals functioning as antioxidants with supplementation considerations. Florida Ruminant Nutrition Symposium. January 30-31, 2007. University of Florida, Gainesville, FL, USA. Available at: https://tinyurl.com/y5zh6h3e

Mlcek, J., Borkovcova, M. and Bednarova, M., 2014. Biologically active substances of edible insects and their use in agriculture, veterinary and human medicine - a review. Journal of Central European Agriculture 15: 225-237. https://doi.org/10.5513/JCEA01/15.4.1533

Moyo, M., Amoo, S.O., Ncube, B., Ndhlala, A.R., Finnie, J.F. and Van Staden, J., 2013. Phytochemical and antioxidant properties of unconventional leafy vegetables consumed in southern Africa. South African Journal of Botany 84: 65-71. https://doi.org/10.1016/j. sajb.2012.09.010

Musundire, R., Zvidzai, C.J., Chidewe, C., Samende, B.K. and Chemura, A., 2016. Habitats and nutritional composition of selected edible insects in Zimbabwe. Journal of Insects as Food and Feed 2: 189198. https://doi.org/10.3920/JIFF2015.0083

Musundire, R., Zvidzai, J.C. and Chidewe, C., 2014. Bio-active compounds composition in edible stinkbugs consumed in southeastern districts of Zimbabwe. International Journal of Biology 6: 36-45. http://dx.doi.org/10.5539/ijb.v6n3p36
Pisoschi, A.M. and Negulescu, G.P., 2011. Methods for total antioxidant activity determination: a review. Biochemistry and Analytical Biochemistry 1: 106. https://doi.org/10.4172/2161-1009.1000106

Plaza, C.M., Torres, D. De, Lorena, E., Robert, K., Gerardo, E., Diaz De Torres, L.E., Lücking, R., Vizcaya, M. and Medina, G.E., 2014. Antioxidant activity, total phenols and flavonoids of lichens from Venezuelan Andes. Journal of Pharmacy \& Pharmacognosy Research 2: 138-147.

Prior, R.L., Wu, X. and Schaich, K., 2005. Standardized methods for the determination of antioxidant capacity and phenolics in foods and dietary supplements. Journal of Agricultural and Food Chemistry 53: 4290-4302.

Qasim, M., Aziz, I., Rasheed, M., Gul, B. and Khan, M.A., 2016. Effect of extraction solvents on polyphenols and antioxidant activity of medicinal halophytes. Pakistan Journal of Botany 48: 621-627.

Quispe-fuentes, I., Vega-gálvez, A. and Campos-requena, V.H., 2017. Antioxidant compound extraction from Maqui (Aristotelia chilensis [Mol] Stuntz) berries: optimization by response surface methodology. Antioxidants 6: 10-22. https://doi.org/10.3390/ antiox6010010

Ramamoorthy, P.K. and Bono, A., 2007. Antioxidant activity, total phenolic and flavonoid content of Morinda citrifolia fruit extracts from various extraction processes. Journal of Engineering Science and Technology 2: 70-80.

Rebaya, A., Belghith, S.I., Baghdikian, B., Leddet, V.M., Mabrouki, F., Olivier, E., Kalthoum Cherif, J. and Ayadi, M.T., 2014. Total phenolic, total flavonoid, tannin content, and antioxidant capacity of Halimium halimifolium (Cistaceae). Journal of Applied Pharmaceutical Science 5: 52-57.

Rice-Evans, C. and Packer, L., 2003. Flavonoids in health and disease. CRC Press, Boca Raton, FL, USA, 504 pp.

Rumpold, B.A. and Schlüter, O.K., 2013. Nutritional composition and safety aspects of edible insects. Molecular Nutrition \& Food Research 57: 802-823. http://doi.wiley.com/10.1002/mnfr.201200735

Santos, D.I., Saraiva, J.M.A., Vicente, A.A. and Moldão-Martins, M., 2019. Methods for determining bioavailability and bioaccessibility of bioactive compounds and nutrients. In: Barba, F.J., Saraiva, J.M.A., Cravotto, G. and Lorenzo, J.M. (eds.). Innovative thermal and nonthermal processing, bioaccessibility and bioavailability of nutrients and bioactive compounds. Woodhead Publishing, Cambridge, UK, pp. 23-54. https://doi.org/10.1016/B978-0-12-814174-8.00002-0

Sayyari, Z. and Farahmandfar, R., 2017. Stabilization of sunflower oil with pussy willow (Salix aegyptiaca) extract and essential oil. Food Science \& Nutrition 5: 266-272. http://doi.wiley.com/10.1002/ fsn3.389

Skrovankova, S., Sumczynski, D., Mlcek, J., Jurikova, T. and Sochor, J., 2015. Bioactive compounds and antioxidant activity in different types of berries. International Journal of Molecular Sciences 16: 24673-24706. https://doi.org/10.3390/ijms161024673

Ssepuuya, G., Mukisa, I.M. and Nakimbugwe, D., 2016. Nutritional composition, quality, and shelf stability of processed Ruspolia nitidula (edible grasshoppers). Food Science \& Nutrition 5: 103112. https://doi.org/10.1002/fsn3.369

Stangeland, T., Remberg, S.F. and Lye, K.A., 2009. Total antioxidant activity in 35 Ugandan fruits and vegetables. Food Chemistry 113: 85-91. https://doi.org/10.1016/j.foodchem.2008.07.026 


\section{G. Ssepuuya et al.}

Syukriah, N., 2014. Effect of solvent extraction on antioxidant and antibacterial activities from Quercus infectoria (Manjakani). International Food Research Journal 21: 1067-1073.

Thaipong, K., Boonprakob, U., Crosby, K., Cisneros-Zevallos, L. and Byrne, H.D., 2006. Comparison of ABTS, DPPH, FRAP, and ORAC assays for estimating antioxidant activity from guava fruit extracts. Journal of Food Composition and Analysis 19: 669-675. https://doi. org/10.1016/j.jfca.2006.01.003
Wrolstad, R.E., Acree, T.E., Decker, E.A., Penner, M.H., Reid, D.S., Schwartz, S.J., Shoemaker, C., Smith, D. and Sporns, P., 2005. Handbook of food analytical chemistry. John Wiley \& Sons, Inc., Hoboken, NJ, USA, 606 pp.

Zielinska, E., Kara, M. and Jakubczyk, A., 2017. Antioxidant activity of predigested protein obtained from a range of farmed edible insects. International Journal of Food Science and Technology 52: 306-312. https://doi.org/10.1111/ijfs.13282 